\title{
Same Same But Different? Analysen zur Struktur kollegialer Kooperation unter Lehrkräften im Kontext von Schul- und Unterrichtsentwicklung
}

\author{
Ulrike Hartmann $(\mathbb{D} \cdot$ Dirk Richter $\cdot$ Cornelia Gräsel
}

Eingegangen: 31. März 2020 / Überarbeitet: 19. Oktober 2020 / Angenommen: 26. Oktober 2020 / Online publiziert: 20. November 2020

(C) Der/die Autor(en) 2020

Zusammenfassung In dieser Studie wird ein Fragebogeninstrument zur Erfassung kollegialer Kooperation bei Lehrkräften vorgestellt. Auf der Basis konzeptueller Annahmen zur Kooperation in Kollegien werden zwei Kooperationsformen (Austausch/low cost und Kokonstruktion/high cost) sowie zwei Inhaltsbereiche (Schulund Unterrichtsentwicklung) erfasst. An einer Stichprobe von 773 Lehrkräften aus 16 weiterführenden Schulen mehrerer deutscher Bundesländer wird die Struktur des Instrumentes mittels konfirmatorischer Faktorenanalysen untersucht. Dabei zeigt sich, dass das Modell, in dem Formen und Inhalte kollegialer Kooperation gleichzeitig berücksichtigt werden, die beste Passung zu den erhobenen Daten aufweist. Die kollegiale Zusammenarbeit zwischen Lehrkräften lässt sich angesichts der vorliegenden Ergebnisse als mehrdimensionales Konstrukt beschreiben. Deskriptive Analysen legen in Übereinstimmung mit bisheriger Forschung nahe, dass Aktivitäten zur unterrichtsbezogenen Kokonstruktion eher selten in Kollegien stattfinden, während ein unterrichtsbezogener Austausch häufiger stattfindet. Im Bereich der schulentwicklungsbezogenen Kooperation fallen die Unterschiede zwischen den Kooperationsformen geringer aus. Die Ergebnisse stützen die faktorielle Validität für eine Erfassung kollegialer Kooperation situiert in spezifischen Inhaltsbereichen der Schul- und Unterrichtsentwicklung.

Schlüsselwörter Lehrerinnen und Lehrer · Unterricht · Schulentwicklung ·

Messung · Konfirmatorische Faktorenanalyse

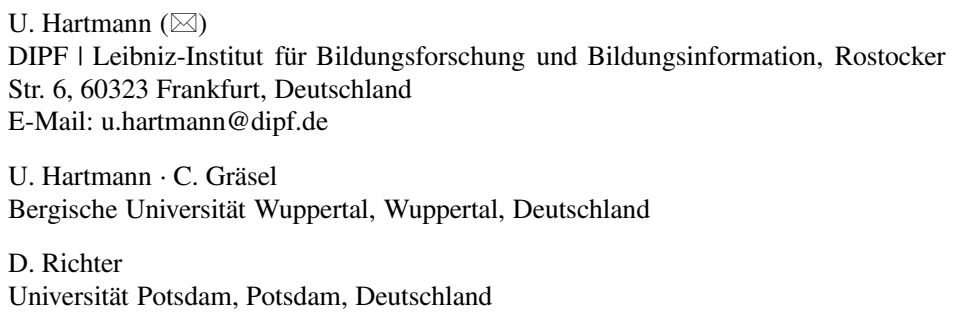




\title{
Same Same But Different? Analyzing the structure of teacher collaboration with regard to contexts of school-related and instructional improvement
}

\begin{abstract}
In this study, we present a survey measure to assess teacher collaboration. Based on existing research, we distinguish two forms (exchange/low cost and coconstruction/high cost) as well as two themes of collaboration (school-related and instructional improvement). In a sample of 773 teachers from 16 high schools from several German federal states, we investigate the structure of the new measure by confirmatory factor analyses. These analyses reveal that a model which simultaneously considers forms and themes of teacher collaboration fits best to the data. Teacher collaboration thus can be described best as a multidimensional construct. In line with previous research, descriptive analyses suggest that high cost activities regarding teachers' instructional practices happen less frequently than low cost instruction-related collaboration. Concerning school development collaboration, differences between the two forms seem to be smaller. These findings imply that teacher collaboration can be assessed as being situated in specific themes for collaborative practices with regard to instruction and school improvement.
\end{abstract}

Keywords Teachers · Cooperation · Instruction · School improvement · Assessment $\cdot$ Confirmatory factor analysis

\section{Einleitung}

Seit mehreren Jahrzehnten beschäftigt sich die Bildungsforschung mit der kollegialen Zusammenarbeit ${ }^{1}$ von Lehrkräften. Für den deutschen Sprachraum wurde die Forschung dazu vor allem durch den 2006 veröffentlichten Themenschwerpunkt in der Zeitschrift für Pädagogik angeregt (Terhart und Klieme 2006). In den letzten Jahren ist erneut - sowohl national als auch international - ein gestiegenes Interesse an der Thematik zu verzeichnen (Fußangel und Gräsel 2014; Grosche et al. 2020; Kolleck 2019; Massenkeil und Rothland 2016; Vangrieken et al. 2015). Ähnliche Tendenzen zeichnen sich in der schulischen Praxis ab: Angesichts aktueller Entwicklungen, beispielsweise durch den Ausbau von Ganztagskonzepten oder der flächendeckenden Umsetzung von Inklusion, erscheint eine kontinuierliche Zusammenarbeit im Kollegium notwendiger denn je. Das lange vorherrschende Bild von der Lehrkraft als Einzelkämpfer*in und der geschlossenen Klassenzimmertür (Lortie 1975) scheint sich allmählich zu wandeln. Das zeigt sich auch darin, dass nahezu alle Lehrkräfte es in einer Befragung von 2016 als wichtig erachten, mit anderen zusammenzuarbeiten (Richter und Pant 2016).

Die empirische Forschung nutzt zur Erfassung der Kooperationsaktivitäten vor allem Fragebögen, in denen Lehrkräfte Angaben darüber machen, wie sie in bestimmten Bereichen ihrer beruflichen Tätigkeit mit anderen zusammenarbeiten. In solchen

\footnotetext{
${ }^{1}$ Die Begriffe „Kooperation“ und „Zusammenarbeit“ stellen laut Duden Synonyme dar. Beide Begriffe werden in diesem Beitrag verwendet.
} 
Fragebögen werden oft zahlreiche Kooperationsaktivitäten erfasst, die sowohl verschiedene Inhaltsbereiche der Zusammenarbeit als auch unterschiedliche Formen kollegialer Kooperation abdecken. Insbesondere die Differenzierung nach verschiedenen Formen (z.B. Austausch, Arbeitsteilung und Ko-Konstruktion) - manchmal auch als Niveaustufen konzipiert - wurde vor allem in der deutschsprachigen Forschung stark rezipiert und bildete die Grundlage für eine Vielzahl empirischer Studien (z. B. Dizinger et al. 2011; Drossel und Willems 2014; Harazd und Drossel 2011; Richter und Pant 2016). Insgesamt ist eine Vielzahl an Operationalisierungen von Kooperationsaktivitäten in Form von Fragebogenskalen zu verzeichnen. Allerdings findet in den vorliegenden Instrumenten keine systematische Differenzierung zwischen unterschiedlichen Inhalten der Kooperation statt, z. B. ob sie der Schul- oder der Unterrichtsentwicklung dienen. An diesem Punkt setzt der vorliegende Beitrag an. Er geht mittels konfirmatorischer Faktorenanalysen der Frage nach, welche Modelle die Kooperationsaktivitäten von Lehrkräften am besten empirisch beschreiben, wenn Kooperationsformen und Kooperationsinhalte gleichzeitig modelliert werden. Dafür nutzt die Studie Fragebogendaten, die in den Jahren 2018 und 2019 an 773 Lehrkräften aus 16 weiterführenden Schulen in Deutschland erhoben wurden. In der folgenden Literaturübersicht werden zunächst mögliche Formen und Inhalte der Kooperation von Lehrkräften beschrieben, bevor anschließend ein neues Messinstrument zur Erfassung kollegialer Kooperation vorgestellt wird, das auf dieser Unterscheidung basiert.

\section{Formen und Inhalte kollegialer Kooperation}

\subsection{Kooperationsformen}

Als Lehrkräftekooperation wird die intentionale Zusammenarbeit mit anderen Personen bezeichnet, die durch einen Bezug auf gemeinsame berufliche Ziele und Aufgaben gekennzeichnet ist (Spieß 2004). Welche Formen Kooperationsbeziehungen unter Lehrkräften annehmen können, ist seit Jahrzehnten Gegenstand theoretischer Modelle. Eine einflussreiche Konzeption stammt von Little (1990), die ausgehend von Lorties soziologischen Studien (1975) ein heuristisches Modell der Lehrkräftekooperation entwickelte. Auf einem Kontinuum stellt sie vier Typen der Kooperation dar, die von storytelling/sharing ideas, materials über aid and assistance bis hin zu joint work reichen. Während der erste Typus Kooperation als reine Weitergabe von Informationen oder Unterrichtsmaterialien beschreibt, wird sie in den weiteren Typen zunehmend reziproker und intensiver im Sinne gemeinsamer Arbeitsaufgaben aufgefasst, bei denen Lehrkräfte sich wechselseitig unterstützen, Einblicke in Arbeitsprozesse ermöglichen und sich in ihrem professionellen Kontext kokonstruktiv weiterentwickeln. Mit dieser Modellvorstellung wird im internationalen Raum bis heute gearbeitet. Auch aktuelle Überblicksarbeiten (z. B. Vangrieken et al. 2015) nutzen ein solches Kontinuum für die Systematisierung kooperativer Tätigkeiten unter Lehrkräften.

Für den deutschen Sprachraum wurden ausgehend von dieser Vorstellung im Jahr 2006 zwei theoretische Modelle vorgestellt. Das am breitesten rezipierte stammt 
von Gräsel et al. (2006a). Es differenziert zwischen drei Kooperationsformen, die in Form individueller Ausprägungen bei Lehrkräften untersucht werden: Austausch, Arbeitsteilung/Synchronisation und Kokonstruktion. Diese implizieren ebenfalls ein zunehmendes $\mathrm{Ma}$ an Reziprozität und der Koordination von Zielstellungen. Im Kontrast zu Littles (1990) Modellvorschlag werden die drei Kooperationsformen von den Autor*innen allerdings nicht auf einem Kontinuum verortet. Sie schließen sich daher weder wechselseitig aus, noch gehen sie vollständig ineinander auf, sondern können je nach Funktion der jeweiligen Arbeitsaufgaben kontextspezifisch parallel innerhalb eines Kollegiums zu finden sein. Die erste Form impliziert einen wechselseitigen Austausch über berufliche Inhalte und Gegebenheiten, ohne dabei eine Zielinterdependenz zwischen den Kooperationspartner*innen einzufordern. Eine solche Art zu kooperieren hat häufig Gelegenheitscharakter und kann insgesamt als low cost-Form der Kooperation betrachtet werden (Gräsel et al. 2006a, S. 210). Die zweite Form wird als arbeitsteilige Kooperation beschrieben. Kern ist dabei die Aufteilung von Aufgaben unter verschiedenen Personen und die Zusammenführung individuell erarbeiteter Ergebnisse. Hierfür ist bereits ein gemeinsames Ziel erforderlich, wobei die arbeitsteilige Ausführung von jeder Person autonom verantwortet wird. Die zentrale Funktion dieser Form von Kooperation liegt nach den Autor*innen in einer Effizienzsteigerung. In der dritten Form, die als Kokonstruktion bezeichnet wird, tauschen sich die Partner*innen intensiv aus und erwerben im wechselseitigen Kontakt miteinander neues Wissen oder konstruieren gemeinsame Problemlösungen. Im Unterschied zur arbeitsteiligen Kooperation werden Aufgaben in dieser Form der Kooperation über weite Strecken gemeinsam bearbeitet, und erfordern damit nicht nur gemeinsame Zielstellungen, sondern auch kontinuierliche Abstimmungen über den Arbeitsprozess. Diese Form der Kooperation wird auch als high cost bezeichnet, da sie sowohl mit einem hohen Aufwand als auch höheren individuellen Risiken für die beteiligten Akteur*innen einhergeht, etwa wenn eigene Vorschläge von anderen kritisiert werden. Beispielhaft führen Gräsel et al. (2006a) die gemeinsame Planung von Unterricht oder das Teamteaching als Indikatoren für eine kokonstruktive Zusammenarbeit an.

Ein weiteres Modell zur Konzeptualisierung von Lehrkräftekooperation schlugen Steinert und Kolleg*innen vor, ebenfalls im erwähnten Themenschwerpunktheft zur Lehrkräftekooperation (Terhart und Klieme 2006). Sie verstehen Kooperation als ein Merkmal, das sich auf der Schulebene verorten lässt, und differenzieren fünf aufeinander aufbauende Niveaustufen von Fragmentierung und Differenzierung über Koordination und Interaktion bis hin zur höchsten Stufe der Integration. Diese Abfolge impliziert auf den unteren Stufen ein mehrheitlich isoliertes und unkoordiniertes Handeln der Lehrkräfte einer Schule, das über mehrere Zwischenstufen zunehmender funktionaler Differenzierung bis hin zu einem ,,systematisch abgestimmten Lehrerhandeln reicht, welches durch wechselseitigen Austausch und Adaption des Handelns der Lehrkräfte charakterisiert ist“" (Steinert et al. 2006, S. 191).

Insbesondere das Modell von Gräsel et al. (2006a) hat vielfältige Untersuchungen angeregt, in denen das selbstberichtete Ausmaß unterschiedlicher Kooperationsformen bei Lehrkräften untersucht wurde. Verschiedene Studien zeigen, dass lockere Austauschkooperationen an Schulkollegien weit verbreitet sind, die engeren kokonstruktiven Formen dagegen nur selten auftreten (z. B. Lang 2009; Gräsel et al. 
2006a). Vergleichsweise aktuelle Erkenntnisse über die Zusammenarbeit von Lehrkräften in Deutschland liefert eine Untersuchung von Richter und Pant (2016), die von infratest dimap an einer repräsentativen Stichprobe von 1015 deutschen Lehrkräften der Sekundarstufe durchgeführt wurde. Das Modell von Gräsel et al. (2006a) wurde in dieser Studie für eine nachträgliche theoretische Einordnung der Befunde herangezogen. Die Autoren stellen fest, dass nahezu alle Lehrkräfte es für wichtig erachten, mit anderen zusammenzuarbeiten. Es zeigen sich jedoch substanzielle Unterschiede im Hinblick auf die theoretisch postulierten Kooperationsformen. Eine deutliche Mehrheit der Lehrkräfte (ca. 80\%) realisiert Kooperationsaktivitäten auf der Ebene des Austausches; auch Aktivitäten, die sich als Arbeitsteilung beschreiben lassen, finden häufig statt (77\%). Eine deutlich geringere Zahl der Lehrkräfte (unter 50\%) gibt an, sich bei Tätigkeiten zu engagieren, die sich als Kokonstruktion beschreiben lassen. Zudem zeigt sich, dass Kooperation nur in Hinblick auf einzelne Tätigkeiten stark ausgeprägt ist. So gaben etwa $60 \%$ der Lehrkräfte an, regelmäßig Lehr- und Unterrichtsmaterial untereinander auszutauschen, jedoch berichteten nur ca. $20 \%$ der Befragten, gemeinsam Projekte zu planen. Das gemeinsame Hospitieren im Unterricht fand nur bei etwa 10\% regelmäßig statt (Richter und Pant 2016). Diese Einschätzungen decken sich mit Ergebnissen weiterer Einzelstudien (z. B. Harazd und Drossel 2011; vgl. Massenkeil und Rothland 2016 für einen Überblick) und werden auch durch international vergleichende Studien bestätigt. Die häufigsten kollaborativen Praktiken, die von Lehrkräften im Rahmen der TALIS-Studie 2013 (Organization for Economic Cooperation and Development 2014) berichtet wurden, waren Materialaustausch und Gespräche über einzelne Schüler*innen. Am wenigsten wurden gemeinsame Arbeitsaktivitäten und gegenseitige Unterrichtshospitationen genannt - Facetten, die wahlweise der Facette Kokonstruktion (Gräsel et al. 2006a) bzw. der Stufe der Integration (Steinert et al. 2006) zuzurechnen wären.

\subsection{Kooperationsinhalte}

Neben den unterschiedlichen Formen, anhand derer Kooperationsaktivitäten in Schulkollegien differenziert werden können, sollten für ein umfangreiches Verständnis von kollegialer Kooperation auch die spezifischen Inhalte der Zusammenarbeit berücksichtigt werden. Orientiert man sich an Modellen aus der Schulentwicklungs- und Schuleffektivitätsforschung (z.B. Ditton 2000; Scheerens 1990), spielt kooperatives Handeln von Lehrkräften in den beiden Kontexten der Unterrichtsund Schulentwicklung eine zentrale Rolle. Die Weiterentwicklung von Unterricht wird in der Forschung als eine professionelle Aufgabe angesehen, für die die möglichst enge und kokonstruktive - Zusammenarbeit von Lehrpersonen als besonders bedeutsam erachtet wird (vgl. Bonsen und Rolff 2006; Gräsel et al. 2006a). Die Unterrichtsentwicklung kann dabei entweder durch neue Anforderungen (z.B. curriculare Vorgaben, Umsetzung von Inklusion, Verwendung digitaler Medien) veranlasst sein (z. B. Grosche et al. 2020) oder der allgemeinen Weiterentwicklung der Unterrichtsqualität dienen, die eine Verbesserung der Schüler*innenleistung nach sich ziehen soll (z. B. Hattie 2012; Sleegers et al. 2014). Seit der Studie von Lortie (1975) wird die Vorbereitung und Durchführung von Unterricht allerdings als Bereich thematisiert, der von Lehrpersonen als private Angelegenheit gesehen 
wird. In den Studien zum Auftreten und zur Intensität von Kooperation zeigt sich dementsprechend, dass unterrichtsbezogenes intensives Zusammenarbeiten relativ selten auftritt bzw. auf unterstützende Bedingungen angewiesen ist (Schütt 2013).

$\mathrm{Zu}$ den konkreten Tätigkeiten, die eine kooperative Unterrichtsentwicklung kennzeichnen, zählen Formen des kollegialen Austausches, beispielsweise die Abstimmung und Information über Bewertungsmaßstäbe und Unterrichtsinhalte durch Lehrkräfte einer Fachgruppe, die Parallelklassen unterrichten. Unterrichtsbezogene Formen von Kokonstruktion stellen beispielsweise fest etablierte Unterrichtsqualitätszirkel in Form von professionellen Lerngemeinschaften oder Hospitationsgruppen dar, sowie Ansätze des Co-Teachings für die kooperative Unterrichtsdurchführung (vgl. Fußangel und Gräsel 2014).

Neben der Bedeutung für die Weiterentwicklung von Unterricht schlägt sich kooperatives Handeln inhaltlich in den Feldern der Schulorganisation und der Professionalisierung des Kollegiums nieder (vgl. Steinert et al. 2006). Kooperative Tätigkeiten umfassen damit beispielsweise den Austausch über schulische Belange außerhalb des eigenen Unterrichts, von der Weitergabe von Informationen aus Konferenzen bis hin zum Austausch über die professionelle Weiterentwicklung im Kollegium. Auch eine gemeinsame Arbeit am Schulprofil, die Vor- und Nachbereitung schulischer Evaluationen sowie kooperationsorientierte Konzepte der Fortund Weiterbildung im Kollegium kennzeichnen eine schulentwicklungsorientierte Kooperation (vgl. Fußangel und Gräsel 2014; Steinert et al. 2006).

Massenkeil und Rothland (2016) weisen auf Basis ihrer Überblicksarbeit auf Wechselwirkungen zwischen Lehrerkooperation und schulischer Entwicklungsarbeit hin. Zum einen setzen Entwicklungsprozesse in der Regel die Kooperation mehrerer Beteiligter voraus, zum anderen können schulische Reformen auch dazu beitragen, kooperative Arbeitsstrukturen an Schulen zu implementieren und tragen damit möglicherweise mittelfristig zu einer erhöhten Innovationskapazität der Einrichtungen bei (Harris et al. 2011; Peurach 2016). Allerdings ist auch hierbei zu berücksichtigen, welche Formen von Kooperationen durch schulische Reformen angeregt werden. Hargreaves und Dawe (1990) haben beispielsweise den Begriff der contrived collegiality (arrangierte Kollegialität) geprägt, die mit einem hohen Ausmaß an Kontrolle durch die Schulleitung und -administration einhergeht. Diese wird allerdings eher nicht langfristig wirksam im Sinne einer kollaborativen und kokonstruktiven Kultur der Offenheit, Unterstützung und des kollegialen Vertrauens an Schulen.

Insgesamt legen sowohl diverse Einzelbefunde als auch systematische Übersichtsarbeiten der letzten Jahre nahe, dass kollegiale Kooperation unter Lehrkräften einen wesentlichen Beitrag sowohl für die Entwicklung der Einzelschule als Organisation als auch für die Unterrichtsentwicklung leisten kann, vor allem wenn an Schulen Kooperationsformen der Kokonstruktion etabliert werden (vgl. Lomos et al. 2011; Massenkeil und Rothland 2016; Vangrieken et al. 2015; Vescio et al. 2008). Es wird allerdings nicht systematisch untersucht, in welchem Verhältnis Kooperationsformen und Inhalte zueinander stehen. 


\section{Messung von Kooperationsaktivitäten in Lehrer*innenkollegien}

Eine Herausforderung für Forschungsarbeiten im Bereich der Lehrkräftekooperation ist die theoretisch begründete und gleichzeitig ökonomische Erfassung dieses facettenreichen Konstruktes. Während in den letzten Jahren mehrere Überblicksarbeiten zu Voraussetzungen, Rahmenbedingungen und Wirkungen kollegialer Kooperation entstanden sind (z.B. Massenkeil und Rothland 2016; Vangrieken et al. 2015; Vescio et al. 2008), finden Aspekte der Operationalisierung deutlich weniger Beachtung. Kolleck (2019) weist auf die große Heterogenität bezüglich der Konzeptualisierung von kollegialer Kooperation hin, die eine Vergleichbarkeit von Studienbefunden erschwert. Für einige Formen der Kooperation unter Lehrkräften, vor allem für Professionelle Lerngemeinschaften, existieren spezifischere Überblicksarbeiten (z. B. Lefstein et al. 2020; Lomos et al. 2011) - diese machen jedoch keine Aussagen zur Struktur unterschiedlicher Kooperationsformen und -inhalte, sondern legen den Fokus in der Regel auf kokonstruktive Praktiken in vorab feststehenden Inhaltsbereichen aus Schul- oder Unterrichtsentwicklung.

Zur Erfassung von Kooperationsaktivitäten ganzer Kollegien haben sich vornehmlich Selbsteinschätzungsskalen als ökonomische Erfassungsmöglichkeit gröBerer Stichproben etabliert. Die Typologie der drei Kooperationsformen Austausch, Synchronisation/Arbeitsteilung und Kokonstruktion von Gräsel et al. (2006a) hat eine große Zahl von Studien im deutschen Sprachraum angeregt, die diese Formen als Ausgangspunkt für eine Operationalisierung von Lehrkräftekooperation verwenden (z. B. Dizinger et al. 2011; Fußangel 2008; Harazd und Drossel 2011; Richter und Pant 2016; Schröder-Lausen und Nerdel 2008). Ein Großteil dieser Studien nimmt dabei die von Gräsel et al. (2006a) vorgeschlagene Einteilung als gegeben an, und verweist auf zufriedenstellende interne Konsistenzen der drei Teilbereiche. Die hohen Interkorrelationen der drei theoretisch angenommenen Faktoren, auf die bereits die Begründer*innen dieser Einteilung 2006 hingewiesen haben, werden allerdings eher selten beleuchtet.

Nur vereinzelt werden faktorenanalytische Prüfungen vorgenommen, die Aufschluss über die empirische Struktur zulassen. Eine Zusammenschau dieser Studien zeigt ein relativ uneinheitliches Bild. Gräsel et al. (2006b) analysieren die Daten von 109 Lehrkräften aus dem Unterrichtsentwicklungsprojekt Chemie im Kontext. Eine explorative Faktorenanalyse legt eine Ein-Faktor-Lösung nahe. Konfirmatorische Faktorenanalysen weisen allerdings auf eine bessere Passung des theoretisch postulierten Drei-Faktorenmodells hin, wobei substanzielle Nebenladungen bestehen, die vor allem die Interpretation der Kooperationsform Synchronisation/Arbeitsteilung erschweren. Die explizite Modellierung dieser Kooperationsform trägt zudem nur noch unwesentlich zur Aufklärung von Varianz in den untersuchten Daten bei. Inhaltlich ist zu berücksichtigen, dass die Itemformulierungen stark an den Projektkontext angelehnt sind, in dem in kollegialen Fachgruppen Unterrichtsentwürfe entwickelt wurden. Die Formulierungen bilden demnach ein etwas eingeschränktes Spektrum kollegialer Kooperation zwischen Lehrkräften ab. Studien, in denen auf Basis der theoretischen Annahmen neu entwickelte Items zum Einsatz kamen, die über die kooperative Weiterentwicklung von Unterricht in Fachgruppen hinausgehen (Drossel und Willems 2014; Kullmann 2010), legen ebenfalls keine eindeutige Ent- 
scheidung bezogen auf die Struktur der Kooperationsformen nahe, auch wenn ein Drei- bzw. Zweifaktorenmodell (Austausch und Kokonstruktion) relativ gesehen die beste Passung zu empirischen Daten aufzuweisen scheint. Zu berücksichtigen sind im Vergleich dieser Studien die durchaus heterogenen Kontexte, die von Entwicklungsprojekten mit Elementen des Design-Based-Research und hohem Commitment der beteiligten Lehrkräfte bis hin zu Survey-Befragungen mit teilweise geringem Rücklauf (unter $30 \%$ bei Drossel und Willems 2014) reichen. Dies erschwert die Vergleichbarkeit von Befunden hinsichtlich der Struktur kollegialer Kooperation in Kollegien.

Neben den Ansätzen, die Kooperationsaktivitäten auf Ebene der Lehrkräfte erfassen, fokussiert das von Steinert et al. (2006) entwickelte Instrument zur Erfassung von Niveaustufen der Kooperation die Schulebene. Es enthält eine Palette von Items zu Unterrichts-, Schulorganisations- und Personalmanagement-Aspekten, die sich auf einer rasch-skalierten Dimension verorten lassen. Die Autor*innen konzipieren kollegiale Kooperation als ein Merkmal von Schulqualität (siehe Abschn. 1.1) und legen den Fokus somit explizit auf die Ebene der Einzelschule. Eine Schule erreicht eine bestimmte Kooperationsstufe dann, wenn mindestens $50 \%$ ihrer Lehrkräfte die zugehörigen Items zustimmend beantworten. In diesem Sinne sind auch die Itemformulierungen zu interpretieren, die teilweise stärker die Rahmenbedingungen für Kooperation abbilden (Für Teamarbeit stehen Arbeitsräume mit ausreichender Ausstattung zur Verfügung oder Beim Entwurf des Stundenplans werden Gelegenheiten zur Teamarbeit berücksichtigt) als tatsächliches Kooperationsverhalten der Lehrkräfte. Bezogen auf die Inhalte von Kooperation werden sowohl Aspekte der Unterrichtsentwicklung (Gegenseitige Unterrichtsbesuche sind ein selbstverständlicher Teil unserer Arbeit; Niveaustufe 4: Integration) als auch der Schulentwicklung (Wir erarbeiten gemeinsam das Profil unserer Schule; Niveaustufe 1: Differenzierung) abgedeckt. Verschiedene Kooperationsinhalte sind dabei auf verschiedenen Stufen ungleich verteilt. Es bleibt offen, inwieweit sich dieser Ansatz und die empirischen Befunde auf das Kooperationsverhalten auf die Lehrkraftebene übertragen lassen.

Betrachtet man die Befunde und methodischen Spezifika der vorgestellten Operationalisierungen über Fragebogenmaße in der Zusammenschau, entsteht ein etwas unscharfes Bild der Erfassung von Kooperationsaktivitäten in Lehrerkollegien. Aufgrund der unterschiedlichen Schwerpunktsetzungen auf Schul- beziehungsweise Unterrichtsentwicklungsaspekte sind die Kontexte nicht immer vergleichbar. Dies erschwert die Interpretation, welche Verhaltensweisen und Aktivitäten kooperatives kollegiales Handeln im schulischen Kontext kennzeichnen. Ferner sind die Befunde zur faktoriellen Struktur kollegialer Kooperation relativ heterogen, auch wenn sich Hinweise auf eine gelungene Differenzierung zwischen Austausch und Kokonstruktion feststellen lassen. Die Analyse unterschiedlicher Formen von Kooperation bildet insofern auch den zentralen Auswertungsfokus vieler der vorgestellten Studien ab. Eine weiterführende Analyse im Hinblick auf unterschiedliche Kooperationsinhalte der Schul- und Unterrichtsentwicklung wurde bislang nicht systematisch umgesetzt. 


\section{Ziele der Studie}

In dieser Studie wird ein Instrument vorgestellt, welches zwei verschiedene Formen (Austausch und Kokonstruktion) sowie zwei verschiedene Inhalte (Schul- und Unterrichtsentwicklung) simultan abbildet. Wir unternehmen damit den Versuch einer vergleichsweise breiten Erfassung kooperativer Tätigkeiten im schulischen Alltag mithilfe eines Fragebogeninstrumentes, das sich im Kontext größerer Kollegiumsbefragungen einsetzen lässt. Die vorliegende Studie orientiert sich dabei an dem Modell von Gräsel et al. (2006a) mit dem Ziel, individuelle Einschätzungen von Lehrkräften bezüglich ihrer Kooperationsaktivitäten zu erfassen.

Die durchgeführten Analysen sollen zudem Aufschluss über die Struktur kollegialer Kooperation ermöglichen. Wir untersuchen anhand des neu entwickelten Instrumentes die Dimensionalität kollegialer Kooperation. Dabei nähern wir uns anhand von Befragungsdaten einer größeren Stichprobe von Lehrkräften weiterführender Schulen der Frage, ob sich kollegiale Kooperationsaktivitäten hinsichtlich ihrer Formen und Inhalte trennscharf voneinander abgrenzen lassen.

\section{Methode}

\subsection{Durchführung und Stichprobe}

Die Datenerhebung fand im Kontext des Projektes CoMMiT (Kooperation an Schulen, Innovation im Team) statt. Das Projekt wurde von der Robert Bosch Stiftung und der Deutschen Schulakademie gefördert. Es handelte sich um die Eingangsbefragung des Projektes, in der alle Lehrkräfte an ihrer Schule im Rahmen einer obligatorischen Lehrerkonferenz einen paper pencil Fragebogen zu ihren Kooperationsaktivitäten ausfüllten. Die Erhebung fand an 16 Schulkollegien weiterführender Schulen aus den Bundesländern Brandenburg, Hessen, Niedersachsen, NordrheinWestfalen und Schleswig-Holstein statt. Alle Schulen entschieden sich freiwillig für eine Projektteilnahme, die neben der schriftlichen Eingangsbefragung weitere Teilstudien zu Aspekten der kollegialen Kooperation sowie ein Fortbildungsangebot umfasste und sich über ca. eineinhalb Schuljahre erstreckte. Die teilnehmenden Schulen in diesem Projektkontext brachten somit ein großes Interesse an schulischer Entwicklungsarbeit und an der Verbesserung kollegialer Kooperationen mit, welches sie zur Teilnahme an dem Gesamtprojekt motivierte.

Die Datengrundlage für die nachfolgenden Analysen besteht aus $N=773$ Lehrkräften (Durchschnittsalter: 43 Jahre; $S D=10,94$ ). Von den Befragten sind 61,9\% weiblich. Durchschnittlich nahmen $70 \%$ der Lehrkräfte eines Kollegiums an der Befragung teil (Spannweite zwischen 35 und 96\%).

\subsection{Instrument}

Das neu entwickelte Instrument zur Erfassung der Lehrkräftekooperation beinhaltet 12 Items zu kollegialen Kooperationsaktivitäten. Lehrkräfte wurden gebeten, auf einer vierstufigen Likert-Skala anzugeben, wie häufig sie verschiedene Aktivitäten an 
Abb. 1 Getestete Modelle

\section{MODELL 1}

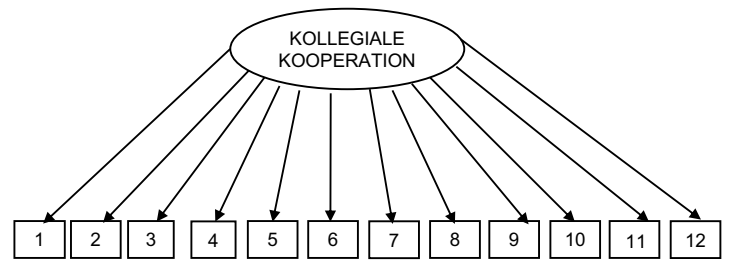

MODELL 2

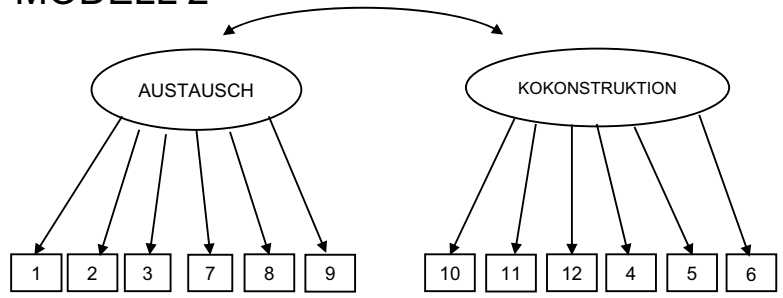

MODELL 3
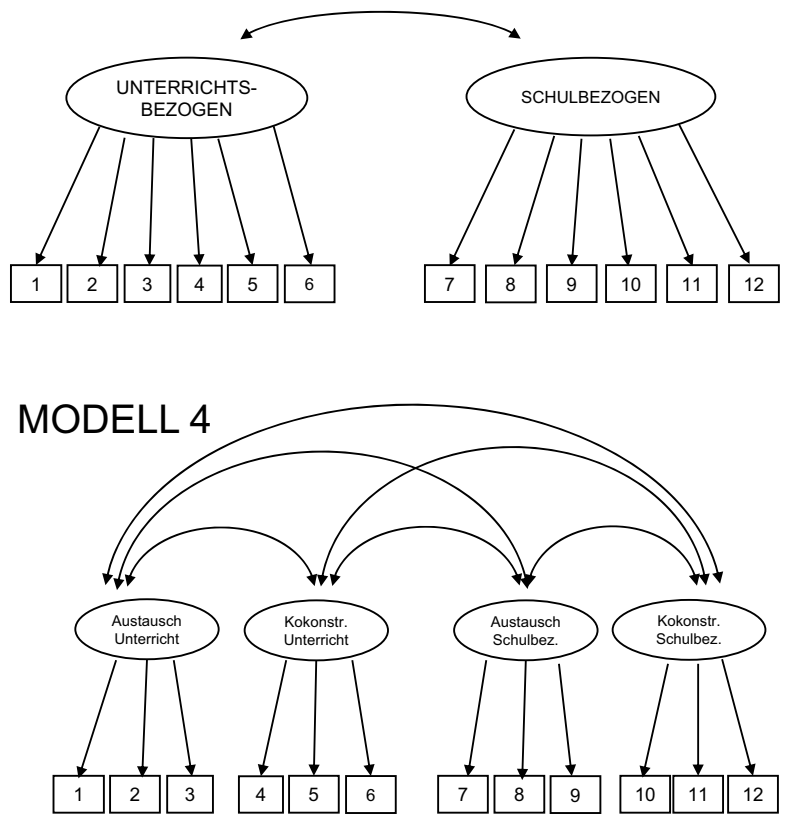
ihrer Schule selbst durchführen ( 1 =nie; 2 = selten; 3 =gelegentlich; $4=$ häufig). In den Itemformulierungen wurden sowohl die beiden Kooperationsformen Austausch und Kokonstruktion als auch zwei verschiedene Inhalte der Kooperation (unterrichtsbezogen und schulbezogen) abgebildet.

Die Skala Unterrichtsbezogene Kooperation besteht aus sechs Items (Cronbachs $\alpha=0,69$ ). Austauschkooperation wurde durch Items wie Ich tausche Unterrichtsmaterialien mit KollegInnen aus abgebildet. Für die Erfassung kokonstruktiver Kooperation wurden Formulierungen wie Ich diskutiere mit KollegInnen über meine Unterrichtsentwürfe gewählt.

Die Skala Schulbezogene Kooperation besteht aus sechs Items $(\alpha=0,74)$. Schulbezogene Austauschkooperation wurde durch Formulierungen wie Ich gebe Informationen aus Konferenzen an KollegInnen weiter operationalisiert; intensivere kokonstruktive Kooperation wurde durch Items wie Ich beteilige mich mit eigenen Beiträgen an der Entwicklung von Konzepten an unserer Schule abgebildet. Das Gesamtinstrument ist in Tab. 1 dargestellt.

\subsection{Analysen}

Zur Beantwortung der Forschungsfragen wurden Konfirmatorische Faktorenanalysen (CFA) mit dem Programm MPlus durchgeführt, um die Struktur des Instrumentes anhand theoretischer Vorannahmen zu prüfen. Es wurden mehrere Modelle geschätzt (MLR; Maximum Likelihood Robust), die im Einklang mit theoretischen Vorannahmen zur Struktur kollegialer Kooperation stehen (siehe Abb. 1): ein Zwei-Faktorenmodell mit den Faktoren Austausch und Kokonstruktion; ein weiteres Zwei-Faktorenmodell mit den Faktoren Schulbezogene Kooperation und Unterrichtskooperation sowie ein Modell mit vier Faktoren (unterrichtsbezogener Austausch, unterrichtsbezogene Kokonstruktion, schulbezogener Austausch, schulbezogene Kokonstruktion). Zum Vergleich wurde ein Ausgangsmodell aufgenommen, das kollegiale Kooperation als eindimensionales Konstrukt modelliert, auf dem alle Items des Instrumentes laden. Diese vier Modelle wurden gegeneinander getestet und hinsichtlich der üblichen Fit-Indizes verglichen. ${ }^{2}$

\section{Ergebnisse}

\subsection{Deskriptiva}

Deskriptive Statistiken für die einzelnen Items des Instrumentes zur Erfassung kollegialer Kooperation sind in Tab. 1 dargestellt. Hinsichtlich der Häufigkeit der berichteten Kooperationsaktivitäten sind Unterschiede festzustellen. Items zur unter-

\footnotetext{
${ }^{2}$ Folgende Kriterien werden zur Einordnung der Modellgüte herangezogen (Bentler und Bonett 1980; Schermelleh-Engel et al. 2003): CFI-und TLI-Werte von >0,90 wird als Hinweis auf einen akzeptablen Fit interpretiert; CFI und TLI $>0,95$ indizieren einen guten Fit. Bezüglich des RMSEA wurde ein Wert $<0,06$ als Hinweis auf einen guten Fit interpretiert, wohingegen SRMR-Werte von $<0,05$ als gut bewertet wurden. Zusätzlich berichten wir AIC und BIC Werte zum Vergleich der relativen Güte der Modelle, da nicht alle ineinander genestet sind (vgl. auch Kumlander et al. 2018).
} 


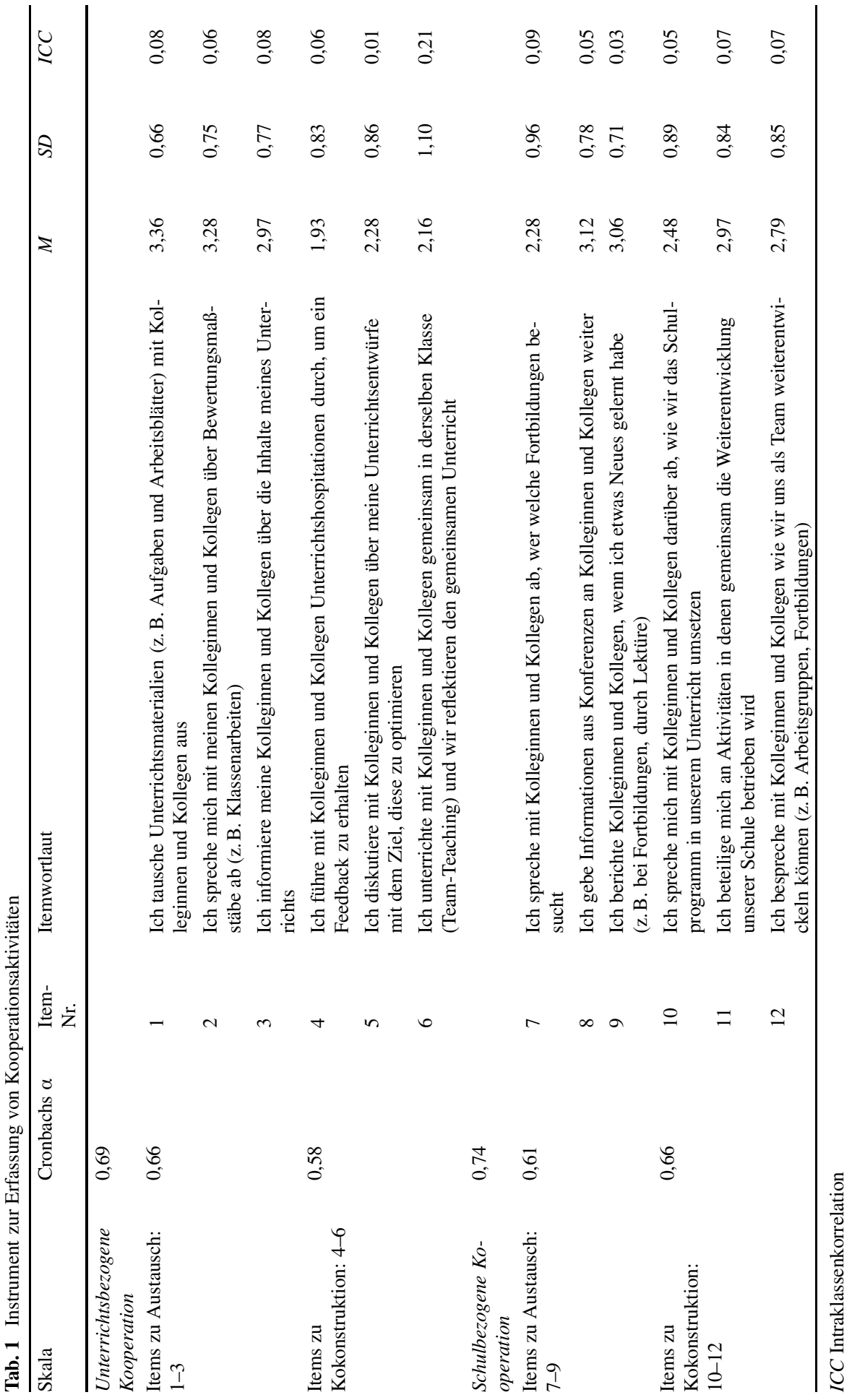


Tab. 2 Interkorrelationsmatrix der Items zur Erfassung kollegialer Kooperation

\begin{tabular}{llllllllllll}
\hline Item & 1 & 2 & 3 & 4 & 5 & 6 & 7 & 8 & 9 & 10 & 11 \\
\hline 1 & - & - & - & - & - & - & - & - & - & - & - \\
2 & 0,48 & - & - & - & - & - & - & - & - & - & - \\
3 & 0,36 & 0,36 & - & - & - & - & - & - & - & - & - \\
4 & 0,24 & 0,25 & 0,23 & - & - & - & - & - & - & - & - \\
5 & 0,31 & 0,32 & 0,38 & 0,46 & - & - & - & - & - & - & - \\
6 & 0,24 & 0,12 & 0,23 & 0,30 & 0,25 & - & - & - & - & - & - \\
7 & 0,22 & 0,22 & 0,26 & 0,17 & 0,17 & 0,13 & - & - & - & - & - \\
8 & 0,28 & 0,29 & 0,24 & 0,17 & 0,15 & 0,14 & 0,33 & - & - & - & - \\
9 & 0,24 & 0,23 & 0,28 & 0,19 & 0,23 & 0,14 & 0,36 & 0,38 & - & - & - \\
10 & 0,12 & 0,20 & 0,26 & 0,23 & 0,28 & 0,18 & 0,26 & 0,22 & 0,22 & - & - \\
11 & 0,12 & 0,15 & 0,28 & 0,23 & 0,15 & 0,20 & 0,27 & 0,32 & 0,32 & 0,36 & - \\
12 & 0,29 & 0,24 & 0,33 & 0,23 & 0,24 & 0,21 & 0,35 & 0,36 & 0,36 & 0,42 & 0,42 \\
\hline
\end{tabular}

Alle $r$ signifikant mit $p<0,01$

richtsbezogenen Kokonstruktion weisen vergleichsweise geringe Häufigkeiten auf (Mittelwerte zwischen 1,93 und 2,28), während Items zum unterrichtsbezogenen Austausch mit Mittelwerten im oberen Bereich der Skala (2,97 bis 3,36) rangieren. Zudem geht aus Tab. 1 hervor, dass die Intraklassenkorrelationen der Items relativ gering ausfallen. Der mittlere Varianzanteil, der auf Schulebene zurückzuführen ist, liegt bei 7,1\% (Spannweite zwischen 0,01 und 0,21).

Darüber hinaus sind aus Tab. 2 die Inter-Item-Korrelationen der untersuchten Stichprobe abzulesen. Die Koeffizienten zwischen den Items des Instrumentes sind alle signifikant und rangieren im geringen bis mittleren Bereich $(0,12<r<0,48)$. Korrelationen über 0,4 lassen sich ausschließlich innerhalb der Itemgruppe einer Subskala der kollegialen Kooperation feststellen. Tendenziell finden sich höhere Korrelationen eher innerhalb der Inhalte kollegialer Kooperation (schulbezogen vs. unterrichtsbezogen). Das Item 6 (Team Teaching) weist im Vergleich die geringsten Interkorrelationen mit anderen Items derselben Skala auf.

\subsection{Faktorenstruktur des Instrumentes}

\subsubsection{Ein-Faktormodell kollegialer Kooperation (Modell 1)}

Im ersten getesteten Modell wird ein allgemeiner Faktor kollegiale Kooperation modelliert, auf dem alle Items laden. Das Modell weist in der von uns untersuchten Stichprobe keine akzeptable Passung auf (siehe Tab. 3).

\subsubsection{Zwei-Faktorenmodell Austausch und Kokonstruktion (Modell 2)}

Das Zwei-Faktorenmodell versucht die Daten anhand der theoretisch angenommenen Unterscheidung der beiden Faktoren Austausch und Kokonstruktion auf zwei Dimensionen abzubilden. Wie aus Tab. 3 hervorgeht, weist dieses Modell ebenfalls keine akzeptable Passung zu den empirischen Daten auf. 
Tab. 3 Vergleich der getesteten CFA-Modelle

\begin{tabular}{lllllllll}
\hline Modell & $\chi^{2}$ & df & CFI & TLI & $\begin{array}{l}\text { RMSEA } \\
{[90 \% \text { CI }]}\end{array}$ & SRMR & AIC & BIC \\
\hline 1 & 396,098 & 54 & 0,80 & 0,75 & $\begin{array}{l}0,090 \\
{[0,082,0,099]}\end{array}$ & 0,064 & $20.927,910$ & $21.095,366$ \\
& & & & & & & \\
2 & 317,275 & 53 & 0,82 & 0,77 & $\begin{array}{l}0,087 \\
{[0,078,0,095]}\end{array}$ & 0,061 & $20.888,645$ & $21.060,753$ \\
& & & & & & & \\
3 & 231,439 & 53 & 0,90 & 0,90 & $\begin{array}{l}0,066 \\
{[0,057,0,075]}\end{array}$ & 0,046 & $20.744,355$ & $20.916,463$ \\
& & & & & & & \\
4 & 132,301 & 48 & 0,95 & 0,93 & 0,048 & 0,036 & $20.643,892$ & $20.839,258$ \\
& & & & & {$[0,038,0,057]$} & & & \\
\hline
\end{tabular}

\subsubsection{Zwei-Faktorenmodell unterrichts- und schulbezogene Kooperation (Modell 3)}

Das Zwei-Faktorenmodell bildet die Daten anhand von zwei Inhaltsbereichen kollegialer Kooperation ab (unterrichts- und schulbezogen). Das Modell weist akzeptable bis gute Fit-Indizes auf, wie in Tab. 3 dargestellt. Der RMSEA liegt allerdings bei diesem Modell mit 0,066 über dem empfohlenen Cut-Off.

\subsubsection{Vier-Faktorenmodell (Modell 4)}

Im Vier-Faktorenmodell werden Kooperationsformen und -inhalte miteinander gekreuzt. Daraus resultieren die Faktoren unterrichtsbezogene Austauschkooperation, unterrichtsbezogene Kokonstruktion, schulbezogene Austauschkooperation so-

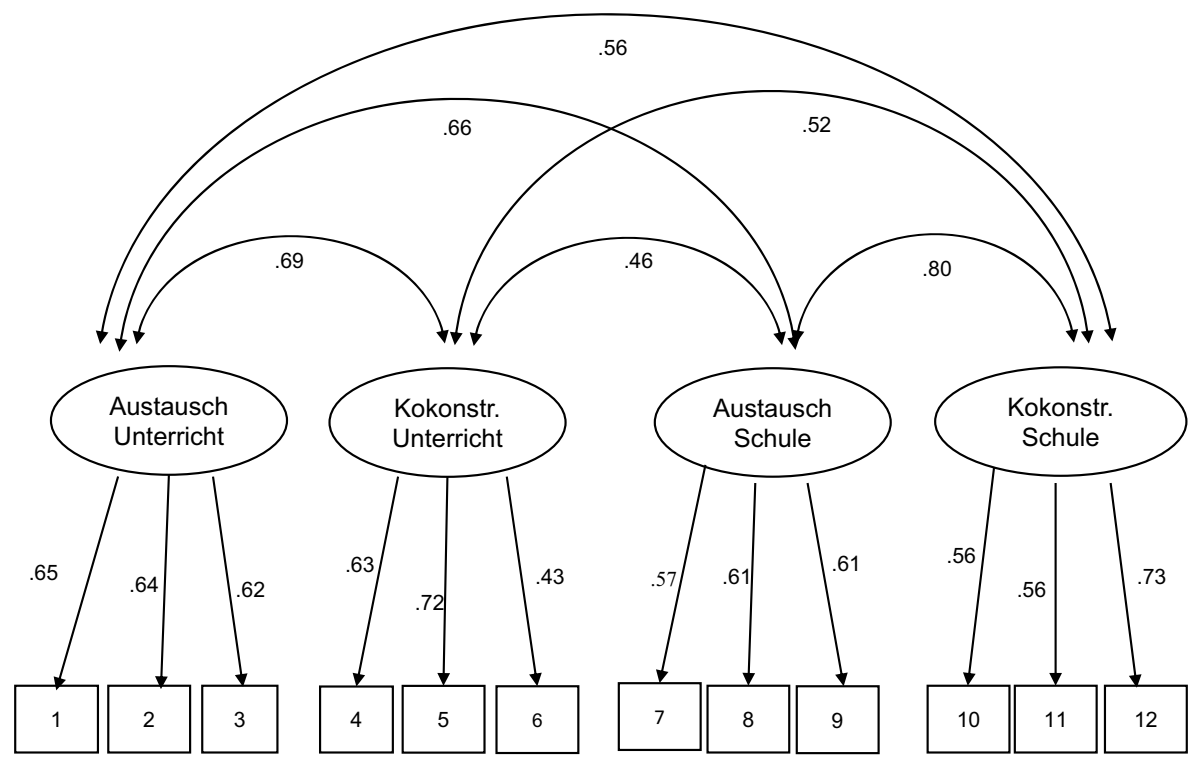

Abb. 2 Faktorstruktur Modell 4. (Die Koeffizienten geben standardisierte Faktorladungen und Korrelationskoeffizienten zwischen den latenten Variablen an) 
wie schulbezogene Kokonstruktion. Dieses Modell weist eine gute Passung zur Datenstruktur auf. Lediglich der Tucker-Lewis-Index verfehlt mit 0,93 knapp den Cut-Off für einen guten Modellfit. Diese Vier-Faktorenstruktur erweist sich auch im direkten Vergleich der Modelle über den AIC $(20.643,892)$ als das passendste.

Das Modell ist in Abb. 2 mit den zugehörigen standardisierten Faktorladungen und messfehlerbereinigten Korrelationen dargestellt. Die Faktorladungen rangieren zwischen 0,42 und 0,73 (alle $p=0,00$ ). Auffällig ist die vergleichsweise geringe Ladung von Item 6 (Team-Teaching) zum Faktor Unterrichtsbezogene Kokonstruktion.

Die zwei latenten Faktoren jeweils eines Inhaltsbereiches korrelieren miteinander zu $r=0,69$ (Unterricht) bzw. $\mathrm{r}=0,80$ (Schule). Zwischen den latenten Faktoren der austauschbezogenen Kooperation in Schule und Unterricht besteht ebenfalls eine recht hohe Korrelation von $r=0,66$. Diese fällt für kokonstruktive Praktiken in Schule und Unterricht mit $r=0,52$ geringer aus, vergleichbar mit den Korrelationen zwischen unterrichtsbezogenem Austausch und schulbezogener Kokonstruktion $(r=0,56)$. Darüber hinaus lässt sich eine mittlere Korrelation $(r=0,46)$ zwischen unterrichtsbezogener Kokonstruktion und schulbezogener Austauschkooperation feststellen.

\section{Diskussion}

\subsection{Zusammenfassung und Interpretation der Ergebnisse}

Die kollegiale Zusammenarbeit zwischen Lehrkräften lässt sich angesichts der vorliegenden Ergebnisse als ein multidimensionales Konstrukt beschreiben. Im Unterschied zu den anderen geprüften Modellen erweist sich eine Struktur als passend, in der unterrichts- und schulbezogene Kooperationshandlungen voneinander unterschieden werden, und in der gleichzeitig die theoretisch postulierten Kooperationsformen Austausch und Kokonstruktion berücksichtigt werden. Kooperation als eindimensional zu betrachten oder lediglich zwischen zwei verschiedenen Formen zu differenzieren, erscheint im Licht der vorliegenden Analysen unterkomplex. Kollegiale Kooperationsaktivitäten von Lehrkräften lassen sich spezifischer in konkreten Inhaltsbereichen der Schul- und Unterrichtsentwicklung verorten. Wie die in dieser Studie analysierten Modelle nahelegen, scheint eine solche Unterscheidung von Inhalten kollegialer Kooperation bei Lehrkräften mindestens so wichtig zu sein wie die Unterscheidung von Kooperationsformen. Dies wurde durch die Sichtung bisheriger Literatur in diesem Forschungsfeld bereits nahelegt; mit dem vorgestellten Instrument wird eine solche systematische Differenzierung erstmalig empirisch umgesetzt.

Anhand der deskriptiven Daten der vorliegenden Studie ist zudem ablesbar, dass die Kooperationsfacette der kokonstruktiven Unterrichtsentwicklung im Vergleich zu den anderen drei Facetten (unterrichtsbezogener Austausch, schulbezogener Austausch und schulbezogene Kokonstruktion) in der von uns untersuchten Stichprobe am seltensten auftritt - ein Befund, der im Einklang mit theoretischen Vorannahmen steht, und der gleichzeitig die bisherige Forschung um eine Differenzierung hinsichtlich des Auftretens kokonstruktiver Praktiken im Kontext von schul- und 
unterrichtsbezogenen Aktivitäten ergänzt. Kokonstruktive Schulentwicklung scheint an Schulen bereits stärker implementiert zu sein als eine gemeinsame Weiterentwicklung des eigenen Unterrichts. Insgesamt überwiegen auch in der von uns untersuchten Stichprobe in Kollegien weiterführender Schulen relativ deutlich die austauschorientierten Formen der Kooperation, sowohl in Bezug auf schul- als auch auf unterrichtsbezogene Inhalte. Dies deckt sich mit Ergebnissen aus einer repräsentativen Fragebogenerhebung (Richter und Pant 2016), und ist darüber hinaus auch angesichts unserer Stichprobe innovations- und kooperationsorientierter Schulen ein durchaus bemerkenswerter Befund, der auf ein bislang wenig genutztes Entwicklungspotential an weiterführenden Schulen hinweisen könnte.

Bei der Weiterarbeit an der Erfassung kollegialer Kooperation sind auf Basis der vorliegenden Studie die substanziellen Interkorrelationen der einzelnen Facetten zu berücksichtigen, die in ihrer Relation durchaus mit theoretischen Vorannahmen korrespondieren (geringere Korrelationen zwischen konzeptuell weiter voneinander entfernten Facetten wie beispielsweise unterrichtsbezogene Kokonstruktion mit schulbezogenem Austausch), allerdings im Gesamtbild doch relativ eng miteinander verwoben sind. Kooperationsaktivitäten werden von Lehrkräften somit nicht primär als Austausch vs. Kokonstruktion wahrgenommen, sondern sind in den spezifischen Inhaltsbereichen der Unterrichts- oder Schulentwicklung situiert, in denen jeweils unaufwändigere Austausch- sowie zeitintensivere Kokonstruktionsformen parallel existieren können, aber nicht müssen.

\subsection{Limitationen}

Einschränkend ist bei der Bewertung der vorliegenden Ergebnisse zu beachten, dass sich das Instrument lediglich auf zwei Formen kollegialer Kooperation bezieht, die theoretisch begründet sind - den Austausch und die Kokonstruktion. Unser Verzicht auf den Aspekt der Synchronisation/Arbeitsteilung liegt nicht in der Annahme begründet, dass wir eine solche Form kollegialer Kooperation ausschließen. Vielmehr liegt hier eine pragmatische Entscheidung der Reduktion auf zwei empirisch gut trennbare Formen kollegialer Kooperation vor, deren Messung sich im Rahmen größerer Fragebogenerhebungen realisieren lässt. Bisherige Forschungsberichte sowie eigene explorative Analysen lassen uns vermuten, dass weitere Kooperationsformen wie die Arbeitsteilung in der schulischen Realität oftmals Handlungsbausteine aus Kokonstruktion und Austausch einschließen, was eine eindeutige Zuordnung auf Itemebene im Rahmen kurzer schriftlicher Befragungen erschwert. Wir möchten jedoch darauf hinweisen, dass qualitative Studien sehr wohl auf die Existenz weiterer Kooperationsformen hinweisen (z. B. Gräsel et al. 2006a; Hartmann und Decristan 2018; Halbheer und Kunz 2011; Maag Merki et al. 2010). Es erscheint nach wie vor möglich, dass in zukünftiger Forschung die Grenzlinien zwischen verschiedenen Formen kollegialer Kooperation soweit expliziert werden, dass deren Erfassung mittelfristig auch im Kontext von Fragebogenerhebungen reliabel und valide realisiert werden kann. Weitere qualitative Studien, die sich spezifisch und theoriebasiert dieser Frage widmen, scheinen angezeigt.

Aus methodischer Perspektive ist einschränkend zu erwähnen, dass die erhobenen Fragebogendaten in einer Mehrebenenstruktur erfasst wurden. Aufgrund der 
Verortung von Lehrkräften in Schulen wäre die hier durchgeführte Analyse auch als Multilevel-Faktorenanalyse denkbar. Dies wäre insbesondere dann erforderlich, wenn ein substanzieller Anteil der Varianz auf Unterschiede zwischen Schulen zurückführbar wäre. In der vorliegenden Studie lag der durchschnittliche Anteil der auf Schulebene befindlichen Varianz bei 7\%. Aufgrund des eher geringen Wertes und aufgrund der geringen Zahl an teilnehmenden Schulen haben wir uns gegen eine Multilevel-Faktorenanalyse entschieden. Simulationsstudien legen zudem nahe, dass das Risiko für eine Parameterverzerrung bei solchen relativ geringen Intraklassenkorrelation auch als eher marginal einzuschätzen ist (Pornprasertmanit et al. 2014). Das Vorgehen in dieser Studie basiert somit auf der Annahme, dass die Beurteilung des eigenen Kooperationsverhaltens von Lehrkräften primär ein individuelles Merkmal darstellt.

Ein weiteres Desiderat bleibt es, den in diesem Beitrag begonnenen Fokus auf die inhaltlichen Facetten kollegialer Kooperation zwischen Lehrkräften noch weiter zu schärfen. So wird in den Analysen des von uns entwickelten Instrumentes ersichtlich, dass die Faktorladung des Team-Teaching-Items, das für die Abbildung von Kokonstruktion unerlässlich erscheint, geringer ausfällt als bei den anderen Items. Auch die Interkorrelationen zu den anderen Items der Skala fallen vergleichsweise gering aus. Eventuell könnte das Label „Team-Teaching“ von Lehrkräften in unterschiedlicher Weise interpretiert worden sein. Eine weitere mögliche Erklärung wäre, dass sich die Umsetzung von Team-Teaching doch stärker auf Schulebene verorten lässt als auf Ebene einzelner Lehrkräfte. Dies liegt zum einen aus inhaltlichen Gründen nahe, da eine Realisierung von Team-Teaching bestimmte Rahmenbedingungen der Schulorganisation voraussetzt, die Lehrkräfte nicht unabhängig von der Schulleitung gestalten können. Zum anderen könnte ein Teilergebnis unserer Studie - der vergleichsweise höhere Varianzanteil des Team-Teaching-Items, der durch die Schulebene aufgeklärt wird - in dieser Art und Weise interpretiert werden. In zukünftigen Erhebungen sollte demnach nicht nur geprüft werden, ob eine einfachere Formulierung im Sinne der gemeinsamen Durchführung und Reflexion von Unterrichtsstunden und der Verzicht auf das spezifische Label „Team-Teaching“ mit besseren Kennwerten innerhalb der Skala der unterrichtsbezogenen Kokonstruktion einhergeht. Es sollte weiterhin der Versuch unternommen werden, die vorgeschlagenen Modelle zu den Kooperationsaktivitäten auf Lehrkraft- wie auf Schulebene stärker miteinander in Beziehung zu setzen, um langfristig ein theoretisch fundiertes Mehrebenenmodell der Lehrer*innenkooperation empirisch prüfen zu können. Mithilfe eines solchen Modells könnten mehrere Einschränkungen dieser Studie gleichzeitig aufgegriffen werden.

Die Ergebnisse, die im Kontext dieser Studie präsentiert werden, sind hinsichtlich ihrer Aussagekraft an die im Projekt CoMMiT untersuchte Stichprobe gebunden. Die Daten lassen beispielsweise keine systematische Aufschlüsselung nach verschiedenen Schultypen zu. Zudem wurden nur Kollegien weiterführender Schulen befragt. Da wir die Befragung an Schulen in mehreren Bundesländern durchgeführt haben, könnte auch auf dieses Merkmal ein gewisser Varianzanteil zurückfallen; systematische Effekte lassen sich in dieser Hinsicht allerdings aufgrund der geringen Schulanzahl pro Bundesland nicht untersuchen. Darüber hinaus können die an der Befragung teilnehmenden Kollegien aufgrund ihrer Verpflichtung für das Ge- 
samtprojekt als vergleichsweise innovations- und kooperationsorientiert eingeschätzt werden, was die Generalisierbarkeit der Ergebnisse auch im Hinblick auf die selbsteingeschätzten Kooperationsaktivitäten in Schulkollegien einschränken könnte. Von einem Selektionseffekt auf Schulebene muss somit ausgegangen werden. Im Vergleich zu vorherigen Studien zur Struktur kollegialer Kooperation ist allerdings zu bemerken, dass wir auf Lehrkraftebene keine starke Selbstselektion der Befragten annehmen müssen, da die Befragungen im Rahmen obligatorischer Konferenzen vor Ort an den teilnehmenden Schulen stattfanden und dadurch eine Beteiligungsquote von durchschnittlich $70 \%$ realisiert werden konnte - wenngleich mit einer relativ hohen Spannweite. Ob sich die an unserer Stichprobe gefundene Struktur auch in anderen Kontexten replizieren lässt, bleibt zum jetzigen Zeitpunkt eine offene Frage, die hoffentlich zu weiterer Forschung im Feld der Lehrkräftekooperation anregen wird.

Als weitere generelle Einschränkung der Aussagekraft dieser Ergebnisse weisen wir auf die prinzipielle Unzulässigkeit hin, Befunde aus Selbsteinschätzungsdaten als validen Indikator für die tatsächlichen Kooperationsaktivitäten von Lehrkräften zu interpretieren. Durch die Verwendung von konkreten Tätigkeitsbeschreibungen in den Items sowie von Ankerformulierungen, die auf die tatsächliche Häufigkeit dieser Tätigkeiten abzielten, haben wir versucht, Kooperationsverhalten möglichst realitätsnah abzufragen. Wir müssen allerdings konstatieren, dass Effekte sozialer Erwünschtheit auch in dieser Studie eine Rolle gespielt haben werden.

\subsection{Ausblick}

Mit dem vorliegenden Beitrag wurde ein Messinstrument zur Erfassung kollegialer Kooperation vorgestellt, das konzeptuell verankert und im Rahmen von Lehrkräftebefragungen relativ ökonomisch einsetzbar ist. Es werden Selbsteinschätzungen bezüglich der Häufigkeit verschiedener Kooperationsformen in den unterschiedlichen Inhaltsbereichen der Schul- und Unterrichtsentwicklung abgebildet, die sich in Strukturanalysen trennscharf voneinander abgrenzen lassen. Die von uns durchgeführten Analysen sprechen für eine Situierung kollegialer Kooperation in diesen Inhaltsbereichen, in denen dann wiederum unterschiedliche Formen der Kooperation (Austausch und Kokonstruktion) realisiert werden können.

Eine weiterführende Frage, die wir nach den durchgeführten Analysen als offen erachten, betrifft die Unterschiedlichkeit kooperativer Arbeitsaktivitäten zwischen unterrichts- und schulentwicklungsbezogenen Kontexten. Während sich im Feld der Unterrichtsforschung mittlerweile eine relativ klare Abgrenzung zwischen einer Austauschkooperation (z.B. im Hinblick auf die Weitergabe von Materialien und Arbeitsblättern) und einer intensiven kokonstruktiven Auseinandersetzung über Unterrichtsentwicklung und -prozesse etabliert hat, scheint dies im Kontext von Weiterentwicklungsprozessen der gesamten Schule zwar auch ein mögliches, aber vielleicht nicht das einzige Unterscheidungsmerkmal zu sein. Kontextspezifische Erkenntnisse über kooperatives Arbeiten von Lehrkräften in Schulentwicklungsprozessen könnten langfristig dazu beitragen, effektive Kooperationsbeziehungen forschungsbasiert fördern zu können. 
Funding Das Projekt CoMMiT, in dem die Daten für diese Studie erhoben wurden, wurde von der Robert Bosch Stiftung gefördert (Projektnummer: 00905930-004). Open Access funding enabled and organized by Projekt DEAL.

Open Access Dieser Artikel wird unter der Creative Commons Namensnennung 4.0 International Lizenz veröffentlicht, welche die Nutzung, Vervielfältigung, Bearbeitung, Verbreitung und Wiedergabe in jeglichem Medium und Format erlaubt, sofern Sie den/die ursprünglichen Autor(en) und die Quelle ordnungsgemäß nennen, einen Link zur Creative Commons Lizenz beifügen und angeben, ob Änderungen vorgenommen wurden.

Die in diesem Artikel enthaltenen Bilder und sonstiges Drittmaterial unterliegen ebenfalls der genannten Creative Commons Lizenz, sofern sich aus der Abbildungslegende nichts anderes ergibt. Sofern das betreffende Material nicht unter der genannten Creative Commons Lizenz steht und die betreffende Handlung nicht nach gesetzlichen Vorschriften erlaubt ist, ist für die oben aufgeführten Weiterverwendungen des Materials die Einwilligung des jeweiligen Rechteinhabers einzuholen.

Weitere Details zur Lizenz entnehmen Sie bitte der Lizenzinformation auf http://creativecommons.org/ licenses/by/4.0/deed.de.

\section{Literatur}

Bentler, P. M., \& Bonett, D. G. (1980). Significance tests and goodness-of-fit in the analysis of covariance structures. Psychological Bulletin, 88, 588-606.

Bonsen, M., \& Rolff, H.-G. (2006). Professionelle Lerngemeinschaften von Lehrerinnen und Lehrern. Zeitschrift für Pädagogik, 52, 167-184.

Ditton, H. (2000). Qualitätskontrolle und Qualitätssicherung in Schule und Unterricht. Ein Überblick zum Stand der empirischen Forschung. Zeitschrift für Pädagogik, 41, 73-92. Beiheft.

Dizinger, V., Fußangel, K., \& Böhm-Kasper, O. (2011). Lehrer/in sein an der Ganztagsschule: Neue Kooperationsanforderungen - neue Belastungen? Zeitschrift für Erziehungswissenschaft, 14, 43-61.

Drossel, K., \& Willems, A. S. (2014). Zum Zusammenhang von Formen der Lehrerkooperation, des Schulleitungshandelns und des Kooperationsklimas an Ganztagsgymnasien. In K. Drossel, R. Strietholt \& W. Bos (Hrsg.), Empirische Bildungsforschung und evidenzbasierte Reformen im Bildungswesen (S. 129-154). Münster: Waxmann.

Fußangel, K. (2008). Subjektive Theorien von Lehrkräften zur Kooperation: Eine Analyse der Zusammenarbeit von Lehrerinnen und Lehrern in Lerngemeinschaften. Wuppertal. http://elpub.bib.uniwuppertal.de/servlets/DerivateServlet/Derivate-1129/dg0802.pdf

Fußangel, K., \& Gräsel, C. (2014). Forschung zur Kooperation im Lehrerberuf. In E. Terhart, H. Bennewitz \& M. Rothland (Hrsg.), Handbuch der Forschung zum Lehrerberuf (S. 846-864). Münster: Waxmann.

Gräsel, C., Fußangel, K., \& Pröbstel, C. (2006a). Lehrkräfte zur Kooperation anregen - eine Aufgabe für Sisyphos? Zeitschrift für Pädagogik, 52, 205-219.

Gräsel, C., Pröbstel, C., Freienberg, J., \& Parchmann, I. (2006b). Anregung zur Kooperation von Lehrkräften im Rahmen von Fortbildungen. In M. Prenzel \& L. Allolio-Nacke (Hrsg.), Untersuchungen zur Bildungsqualität von Schule: Abschlussbericht des DFG-Schwerpunktprogramms (S. 310-329). Münster: Waxmann.

Grosche, M., Fußangel, K., \& Gräsel, C. (2020). Kokonstruktive Kooperation zwischen Lehrkräften: Aktualisierung und Erweiterung der Kokonstruktionstheorie sowie deren Anwendung am Beispiel schulischer Inklusion. Zeitschrift für Pädagogik, 4, 461-479.

Halbheer, U., \& Kunz, A. (2011). Kooperation von Lehrpersonen an Gymnasien. Eine qualitative und quantitative Analyse der Wahrnehmung von Lehrpersonen aus schul- und governancetheoretischer Perspektive. Wiesbaden: VS.

Hargreaves, A., \& Dawe, R. (1990). Paths of professional development: contrived collegiality, collaborative culture, and the case of peer coaching. Teaching and Teacher Education, 6, 227-241.

Harris, A., Dinham, S., \& Crowther, F. (2011). System improvement through collective capacity building. Journal of Educational Administration, 49, 624-636.

Hartmann, U., \& Decristan, J. (2018). Brokering activities and learning mechanisms at the boundary of educational research and school practice. Teaching and Teacher Education, 74, 114-124.

Hattie, J. (2012). Visible learning for teachers: Maximizing impact on learning. London: Routledge. 
Harazd, B., \& Drossel, K. (2011). Formen der Lehrerkooperation und ihre schulischen Bedingungen Empirische Untersuchung zur kollegialen Zusammenarbeit und Schulleitungshandeln. Empirische Pädagogik, 25, 145-160.

Kolleck, N. (2019). Motivational aspects of teacher collaboration. Frontiers in Education. https://doi.org/ 10.3389/feduc.2019.00122.

Kullmann, H. (2010). Lehrerkooperation an Gymnasien: Ausprägung und Wirkungen am Beispiel des naturwissenschaftlichen Unterrichts. Münster: Waxmann.

Kumlander, S., Lahtinen, O., Turunen, T., \& Salmivalli, C. (2018). Two is more valid than one, but is six even better? The factor structure of the Self-Compassion Scale (SCS). PLoS One, 13. https://doi.org/ 10.1371/journal.pone.0207706.

Lang, M. (2009). Intensivierung von Lehrerkooperation durch schulische Innovationsvorhaben zum selbstgesteuerten Lernen? Befunde aus dem BLK-Modellversuchprogramm SKOLA. Zeitschrift für Evaluation, 8, 87-106.

Lefstein, A., Louie, N., Segal, A., \& Becher, A. (2020). Taking stock of research on teacher collaborative discourse: Theory and method in a nascent field. Teaching and Teacher Education. https://doi.org/10. 1016/j.tate.2019.102954.

Little, J. W. (1990). The persistence of privacy: Autonomy and initiative in teachers' professional relations. Teachers College Record, 91, 509-536.

Lomos, C., Hofman, R.H., \& Bosker, R.J. (2011). Professional communities and student achievement-a meta-analysis. School Effectiveness and School Improvement, 22, 121-148.

Lortie, D.C. (1975). Schoolteacher-a sociological study. Chicago: University Press.

Maag Merki, K., Werner, S., Kunz, A., \& Luder, R. (2010). Professionelle Zusammenarbeit in Schulen. Blick über den Zaun: Kooperation an Zürcher Volksschulen. SchulVerwaltung Nordrhein-Westfalen, 21, 334-335.

Massenkeil, J., \& Rothland, M. (2016). Kollegiale Kooperation im Lehrerberuf.: Überblick und Systematisierung aktueller Forschung. In K. Moegling, S. Hadeler \& G. Hund-Göschel (Hrsg.), Was sind gute Schulen? Teil 1. Konzeptionelle Überlegungen und Diskussion (S. 87-118). Immenhausen bei Kassel: Prolog-Verlag.

Organization for Economic Cooperation and Development (2014). TALIS 2013 results: An international perspective on teaching and learning. Paris: OECD.

Peurach, D. J. (2016). Innovating at the nexus of impact and improvement: Leading educational improvement networks. Educational Researcher, 45, 421-429.

Pornprasertmanit, S., Lee, J., \& Preacher, K. J. (2014). Ignoring clustering in confirmatory factor analysis: Some consequences for model fit and standardized parameter estimates. Multivariate Behavioral Research, 49, 518-543.

Richter, D., \& Pant, H. A. (2016). Lehrerkooperation in Deutschland. Eine Studie zu kooperativen Arbeitsbeziehungen bei Lehrkräften der Sekundarstufe I. Gütersloh: Bertelsmann Stiftung, Robert Bosch Stiftung, Stiftung Mercator, Deutsche Telekom Stiftung.

Scheerens, J. (1990). School effectiveness and the development of process indicators of school functioning. School Effectiveness and School Improvement, 1, 61-80.

Schermelleh-Engel, K., Moosbrugger, H., \& Müller, H. (2003). Evaluating the fit of structural equation models: Tests of significance and descriptive goodness-of-fit measures. Methods of Psychological Research-Online, 8, 23-74.

Schröder-Lausen, E., \& Nerdel, C. (2008). Kooperation von Grundschullehrkräften zum Heimat- und Sachunterricht - Erste Ergebnisse einer Fragebogenstudie. Zeitschrift für Didaktik der Naturwissenschaften, 14, 185-200.

Schütt, S. (2013). Kooperation in der Schule: Eine Untersuchung der Orientierungs- und Handlungsmuster von Lehrern. Bern: Peter Lang.

Sleegers, P., Thoonen, E., Oort, J., \& Peetsma, T. (2014). Changing classroom practices: The role of schoolwide capacity for sustainable improvement. Journal of Educational Administration, 52, 617-652.

Spieß, E. (2004). Kooperation und Konflikt. In H. Schuler \& K. H. Sonntag (Hrsg.), Enzyklopädie der Psychologie/Organisationspsychologie - Gruppe und Organisation (S. 193-250). Göttingen: Hogrefe.

Steinert, B., Klieme, E., Maag Merki, K., Döbrich, P., Halbheer, U., \& Kunz, A. (2006). Lehrerkooperation in der Schule: Konzeption, Erfassung, Ergebnisse. Zeitschrift für Pädagogik, 52, 185-204.

Terhart, E., \& Klieme, E. (2006). Kooperation im Lehrerberuf - Forschungsproblem und Gestaltungsaufgabe: Zur Einführung in den Thementeil. Zeitschrift für Pädagogik, 52, 163-166.

Vangrieken, K., Dochy, F., Raes, E., \& Kyndt, E. (2015). Teacher collaboration: A systematic review. Educational Research Review, 15, 17-40.

Vescio, V., Ross, D., \& Adams, A. (2008). A review of research on the impact of professional learning communities on teaching practice and student learning. Teaching and Teacher Education, 24, 80-91. 